\title{
HLA-B27 associated reactive spondyloarthropathies in a Dutch military hospital
}

\author{
R J LIONARONS, M VAN ZOEREN, J N VERHAGEN, AND H A LAMMERS
}

From the Department of Internal Medicine, Militair Hospitaal 'Dr A Mathijsen', J Haydnlaan 2, 3533 AE Utrecht, The Netherlands

SUMMARY Forty-two male patients with reactive spondyloarthropathies in a Dutch military hospital are described with mean age of onset 21.9 years. Peripheral arthritis or sacroiliitis was present in all, eye symptoms in $21(50 \%)$, and genitourinary disease in $15(35 \cdot 7 \%)$. Evidence of antecedent sexually acquired or enterocolitic infection was found only in three $(7 \cdot 2 \%)$. HLA-B27 antigen was detected in $34(81 \%)$ of 42 patients. Additional data suggest that reactive spondyloarthropathies are the most common form of inflammatory arthritis in young men.

Key words: reactive arthritis, seronegative arthritis, Reiter's syndrome, ankylosing spondylitis.

The term 'reactive arthritis' is used to describe acute, non-purulent arthritis that follows a bacterial infection elsewhere in the body. A classic example of reactive arthritis is rheumatic fever, which is triggered by a $\beta$-haemolytic streptococcal infection in the throat.

Classification of reactive arthritis as HLA-B27 associated or non-HLA-B27 associated, and of the former group as sexually acquired, enterocolitic, or of unknown origin can be made. ${ }^{1}$

Here we report our clinical experience with 42 cases of reactive arthritis in a Dutch military hospital during a period of one year.

The purpose of the present study was to establish the prevalence of this heterogeneous clinical entity and to categorise seronegative spondyloarthropathies in a relatively young population.

\section{Patients and methods}

Consecutive unrelated Dutch patients attending the Department of Internal Medicine of the 'Militair Hospitaal Dr A Mathijsen' were selected for study. $99 \%$ of the population served by this hospital is male, and $61 \%$ of all patients attending are aged 35 years or younger. All of them were seen by the principal investigator (RJL) and at least one of the other authors. They were questioned in detail about their joint symptoms, the presence of heel pain, low

Accepted for publication 1 July 1985.

Correspondence to Dr R J Lionarons. De Warande 83, 3972 XW Driebergen-Rijsenburg. The Netherlands. backpain or stiffness, mucosal and skin lesions, symptoms of acute anterior uveitis, conjunctivitis, urethritis, inflammatory bowel disease, and sexual exposure. Their past and family histories of these disorders were also recorded.

A thorough physical examination was performed, with special attention to the joints, spine, skin, eyes, buccal mucosa, and genitalia.

Tests for rheumatoid factor by the standard latex agglutination test and the Waaler-Rose tests, a test for antinuclear antibodies by indirect immunofluorescence, and a microlymphocytotoxicity test to determine HLA-B27, were performed.

Posteroanterior and oblique roentgenograms of the sacroiliac joints were obtained in all patients. The radiographic changes of sacroiliitis were graded from I to III (Table 1).

When intestinal symptoms were present salmonella, shigella, campylobacter, and yersinia infections were excluded by stool cultures and tests for agglutinating antibodies. Diagnosis of Chlamydia trachomatis infection was made from urethral cultures combined with measurements of antibody levels.

Table 1 Radiographic grading of sacroiliitis

Grade I : Loss of clear-cut cortical joint margins with subchondral erosions and apparent widening of joint space

Grade II : Sclerosis of varying degree and extent along the joint margins and narrowing of the joint space

Grade III: Obliteration of the joint space with bony fusion 
The following diagnoses were applied: ankylosing spondylitis according to the Rome criteria ${ }^{2}$ Reiter's disease, based on the occurrence of arthritis with one or more of the extraarticular features typical of this disease (urethritis, balanitis, conjunctivitis, iritis); psoriatic arthritis suggested by the characteristic skin lesions or nail pits or involvement of distal interphalangeal joints; ulcerative colitis or Crohn's disease and arthritis; a remaining group of seronegative spondyloarthropathies that did not fit into any of these well defined categories but were positive for HLA-B27.

\section{Results}

A total of 42 patients with seronegative spondyloarthropathies were seen. All were men. The mean age of onset of disease was 21.9 years (range 11 to 53 ), and in $88.1 \%$ of them the disease started in the second and third decades (Table 2).

Antecedent diarrhoeal illness occurred in six and non-specific urethritis in six patients. Four patients had both symptoms.

All suffered from arthritis. A peripheral arthritis was the presenting feature in $32(76 \cdot 2 \%)$ and sacroiliitis (low back pain with early morning stiffness and sacroiliac joint tenderness) in 10 $(23.8 \%)$. The average number of joints affected in the 42 patients was 3.6 (range one to nine). The number of joints involved was one in five patients, two in eight, three in 11 , four in six, and more than four in 12 patients. The sacroiliac joints and weightbearing joints of the lower extremities were commonly affected (Table 3 ). Eye symptoms were found in $21(50 \%)$ and genitourinary complaints in $15(35 \cdot 7 \%)$. Heel pain was recorded in one patient with radiographic periostitis of the calcaneum. Painless oral ulcers were seen in two and other mucocutaneous lesions, including keratoderma blennorrhagia, nail changes, and circinate balanitis were prominent in six patients. Significant weight

Table 2 Age at onset of reactive spondyloarthropathies in 42 patients in the military hospital

\begin{tabular}{lc}
\hline Age (years) & Number of patients \\
\hline $10-14$ & 3 \\
$15-19$ & 14 \\
$20-24$ & 13 \\
$25-29$ & 7 \\
$30-34$ & 4 \\
$35-39$ & 0 \\
$40-44$ & 0 \\
$45-49$ & 0 \\
$50-54$ & 1 \\
\hline
\end{tabular}

loss had occurred in eight $(19 \%)$ patients (range 6-14 kg) and fever was documented in $14(33 \cdot 3 \%) \frac{\mathrm{O}}{\mathrm{\omega}}$ and ranged from 38.1 to $39.2^{\circ} \mathrm{C}$. Ten patients? $(23.8 \%)$ had a family history of inflammatory $\stackrel{\vec{\rho}}{\vec{F}}$ arthritis, spondylitis, or psoriasis.

Laboratory data showed a significantly raised $\frac{C}{0}$ erythrocyte sedimentation rate (range $22-89 \mathrm{~mm} / 1 \mathrm{st} \frac{\overline{\bar{p}}}{\overline{\frac{p}{2}}}$ h; mean 34) in $22(52.4 \%)$ patients. A micro- $\Phi$ lymphocytotoxicity test showed the presence of HLA-B27 antigen in $34(81 \%)$ of 42 patients. Serum ${ }^{\text {क }}$ uric acid was raised in two patients taking thiazide $\overrightarrow{0}$ diuretics. One patient displayed a positive test for rheumatoid factor on one occasion, but the test was $\omega_{\sigma}$ negative on a subsequent occasion. The same phenomenon occurred in one patient with fluorescent antinuclear antibody tests.

Though only 10 patients had clinical signs of ? sacroiliitis as a presenting symptom, another $14 \mathrm{had} \vec{A}$ radiographic evidence of at least grade II or III $\vec{\circ}$ sacroiliitis during the course of their illness.

Serological evidence of previous yersinia infection $\overrightarrow{-}$ was found in two and positive stool cultures for $\mathbb{D}$ salmonella in one patient.

Diagnoses made before our evaluation had been various: only nine $(21.4 \%)$ patients were suspected of reactive arthritis by their general practitioner $\delta$ by other specialists at their first visit to our hospitat; two were treated for gout with colchicine ame allopurinol, without relief of symptoms; and ones had been labelled as having rheumatic fever, but an infection with $\beta$-haemolytic streptococcus in the throat could not be demonstrated. Rheumatoid $\mathbb{Q}$ arthritis was the referring diagnosis in one, and $\overrightarrow{\overrightarrow{0}}$ undiagnosed polyarthritis or low back pain, or both $\exists$ in 29 patients. We found: ankylosing spondylitis (eight patients); Reiter's disease (22); Crohn'so

Table 3 Frequency of joint involvement in 42 patients with reactive spondyloarthropathies

\begin{tabular}{|c|c|}
\hline Joints involved & Frequency (\%) \\
\hline Thoracic spine & $16 \cdot 7$ \\
\hline Lumbar spine & $14 \cdot 3$ \\
\hline Sacroiliac joint & $57 \cdot 1$ \\
\hline Shoulder & $33 \cdot 3$ \\
\hline Elbow & $19 \cdot 0$ \\
\hline Wrist & $33 \cdot 3$ \\
\hline $\mathrm{MCP}^{*}$ & $16 \cdot 7$ \\
\hline PIP/DIP* & $9 \cdot 5$ \\
\hline Sternocostal joints & $14 \cdot 3$ \\
\hline Knee & $64 \cdot 3$ \\
\hline Hip & $14 \cdot 3$ \\
\hline Ankle & $38 \cdot 1$ \\
\hline MTP* & $28 \cdot 6$ \\
\hline
\end{tabular}


disease with arthritis (one); the remaining group (11).

\section{Discussion}

The present study describes reactive spondyloarthropathies in predominantly young males. Though antecedent enteric or genital infection was suspected in 16 patients $(38 \cdot 1 \%)$ only three proved infections were recorded. In the past the absence of infections delayed diagnosis and in many instances led to inappropriate diagnoses and ineffectual therapy. The discovery of the strongly associated genetic marker HLA-B27 has simplified categorisation. ${ }^{34}$

Two concomitant toxoplasmosis infections with high serum antibody titres, an infection of the throat with haemolytic streptococcus of group $\mathrm{C}$, and one serum hepatitis infection at the onset of disease were not considered to be responsible for the clinical entities that evolved. ${ }^{5}$ Previous trauma, recently described as a possible factor precipitating reactive arthritis, ${ }^{6}$ was seen in one patient who presented with chronic pain, swelling and stiffness of the right knee. He had struck his knee one week before, playing soccer. Knee $x$-rays were normal and arthroscopy showed no intra-articular lesions. Joint fluid cytology and synovial biopsy showed a nonspecific synovitis with marked lymphocyte infiltration. The patient had suffered from arthritis of the left wrist six months previously. As far as we know Noer was the first to conclude in his " $E x$ perimental" epidemic of Reiter's syndrome' that arthritis seemed worse in joints previously weakened by other disease. ${ }^{7}$

The main features of the reactive spondyloarthropathies in our hospital are comparable with those found in other series. ${ }^{8-10}$ They include oligo- or polyarthritis with prominent involvement of the lower limb, sacroiliitis, eye symptoms, and genitourinary infections. Only a few had enthesopathy or mucocutaneous lesions. All but two patients were seronegative. Both positive rheumatoid factor and antinuclear antibody tests were negative on a subsequent occasion. Two patients with raised uric acid levels did not respond to the usual therapies for gout. HLA-B27 was present in all of them and in a large proportion of our group $(81 \%)$. Keat recently presented collected results of comparable studies from different countries. ${ }^{11}$ He found HLA-B27 in $79.4 \%$ of patients with shigella arthritis, $83.7 \%$ in salmonella arthritis, $72 \cdot 2 \%$ in campylobacter arthritis, $74.9 \%$ in yersina arthritis, and $79.5 \%$ in patients with sexually acquired reactive arthritis (mean $77 \cdot 9 \%$ ).

HLA-B27 associated reactive spondyloarthropathies may be far more prevalent than previously
Table 4 Causes of inflammatory arthritis in young men aged 15 to 35 years" at the Militair Hospitaal 'Dr A Mathijsen', April 1982-March 1983

\begin{tabular}{lc}
\hline Disorder & Number of patients \\
\hline Reiter's syndrome & 21 \\
Ankylosing spondylitis & 6 \\
Crohn's arthritis & 1 \\
Reactive arthritis remaining group & 10 \\
Systemic lupus erythematosus & 1 \\
Septic arthritis & 2 \\
Sarcoid arthritis & 3
\end{tabular}

*This age range excludes four of the patients included in Table 2 and includes a further six patients with other diagnoses.

suspected. ${ }^{12}$ Comparative figures for other rheumatic diseases in our hospital are shown in Table 4.

It is of great importance that no cases of rheumatoid arthritis, the most common inflammatory arthropathy, ${ }^{13}$ were seen, while 38 of $44(86.4 \%)$ patients had some form of reactive arthritis. However, it should be noted that a military hospital will care for a larger aggregation of young men than a civilian institution. Therefore, reactive arthritis should be the first diagnosis to be considered in young men presenting with seronegative spondyloarthropathies.

We thank Dr J W Imhof for valued discussions.

\section{References}

1 Keat A C. Scott J T. Ridgway G L. Maini R N. Pegrum G D. Sexually acquired reactive arthritis. Ann Rheum Dis 1979: 38 (suppl 1): 52-4.

2 Kellgren J H, Jeffrey M R. Ball J, eds. The epidemiology of chronic rheumatism. Oxford: Blackwell. 1963: 1: 326.

3 Joliat G. Ferro A. Jeannet M. Ott H. HLA-B27 antigen in diagnosis of atypical seronegative inflammatory arthropathy. Ann Rheum Dis 1976; 35: 531-3.

4 Keat A C. Barnes R M R. HL-A27-associated arthritis. Rheumatol Rehabil 1976; 15: 87-91.

5 Duffy P, Lidsky M D. Sharp J T. et a!. Polyarthritis. polyarteritis and hepatitis B. Medicine (Baltimore) 1976: 55: 19-37.

6 Wisnieski J J. Trauma and Reiter's syndrome: development of reactive arthropathy in two patients following musculoskeletal injury. Ann Rheum Dis 1984: 43: 829-32.

7 Noer H R. An 'experimental' epidemic of Reiter's syndrome. JAMA 1966; 198: 693-8.

8 Arnett F C. Incomplete Reiter's syndrome: clinical comparison with classical triad. Ann Rheum Dis 1979: 38 (suppl 1): 73-8.

9 McClusky O E. Lordon R E. Arnett F C. HL-A27 in Reiter's syndrome and psoriatic arthritis. J Rheumatol 1974; 1: 263-8.

10 Ford D K. Reiter's syndrome. Bull Rheum Dis 1970; 20: 588-91.

11 Keat A C. Reiter's syndrome and reactive arthritis in perspective. $N$ Engl J Med 1983; 309: 1606-15.

12 Arnett F C. McClusky O E, Schacter B Z. Lordon R. Incomplete Reiter's syndrome: discriminating features and HLAw27 in diagnosis. Ann Intern Med 1976; 84: 8-12.

13 Nuki G, Brooks R, Buchanan W W. The economics of arthritis. Bull Rheum Dis 1973; 23: 726-33. 